Stud. Univ. Babeş-Bolyai Math. 66(2021), No. 4, 605-611

DOI: 10.24193/subbmath.2021.4.01

\title{
On a property of the generalized Brauer pairs
}

\author{
Tiberiu Coconeț
}

\begin{abstract}
In this paper we give a generalization of a result of Puig and Zhou to the context of group graded algebras. We use this generalization for an alternative approach of the proof of a result involving group graded basic Morita equivalences.
\end{abstract}

Mathematics Subject Classification (2010): 20C20, $20 \mathrm{C} 05$.

Keywords: Blocks, Brauer pairs, defect groups, normal subgroups, graded algebras, permutation algebras.

\section{Introduction}

The extended Brauer quotient for $G$-interior algebras was first introduced in [5]. The construction is useful for extending to the blocks of the normalizers of the local pointed groups the local equivalences induced by a basic Morita equivalence. The construction was then generalized in [4] to the case of $H$-interior $G$-algebras, where $H$ is normal in $G$. Further remarks and properties are given in [2] and [3] for the case of $\mathrm{G} / \mathrm{H}$-graded algebras, serving for the generalization of the main rezult of [5], which was achived in [3].

In the current paper we generalize [6, Lemma 4.18] to the case of graded algebras. We obtain this generalization by employing a generalized Brauer pair and we first state and prove, without referring to graded algebras, Theorem 4.2. Secondly we give Corollary 4.4. This approach is necessary due to some technical difficulties involving the blocks of the generalized Brauer pairs. On the other hand the mentioned generalization can be used for giving an alternative proof for the main result of [2]. We discuss these situations in section 4.

In Sections 2 and 3 we introduce the standard notations and the basic results needed for the main result.

This work was supported by a grant of the Romanian Ministry of Education and Research, CNCS UEFISCDI, project number PN-III-P1-1.1-TE-2019-0136, within PNCDI III. 


\section{Assumptions and general settings}

Throughout this paper $\mathcal{O}$ denotes a discrete complete valuation ring with $k:=$ $\mathcal{O} / J(\mathcal{O})$ its residue class field, of characteristic $p . G$ is always a finite group such that $p$ divides its order. All modules and algebras are finitely generated or of finite dimension. A $G$-algebra over $\mathcal{O}$ is an algebra $A$ such that there exists a group homomorphism $G \mapsto \operatorname{Aut}_{\mathcal{O}}(A)$ compatible with the algebra structure of $A$. For any $a \in A$ and any $g \in G$ we denoted by $a^{g}$ the result of $g$ acting on $a$.

A $G$-interior algebra over $\mathcal{O}$ is an algebra $A$ endowed with a group homomorphims $G \rightarrow A^{*}$ such that $g \mapsto g \cdot 1_{A}=1_{A} \cdot g$, where $A^{*}$ denotes the units of $A$. Any $G$-interior algebra is becomes a $G$-algebra via conjugation with the elements of $G$. Explicitly for any $a \in A$ and $g \in G$ we have $a^{g}=g^{-1} \cdot a \cdot g$.

For all subgroups $K$ in $G$, on a $G$-algebra $A$, we denote by $A^{K}$ the set of all $K$-fixed elements of $A$. If $L$ is also a subgroup of $G$ with $L \leq K$ then the $\mathcal{O}$-module homomorphism

$$
\operatorname{Tr}_{L}^{K}: A^{L} \rightarrow A^{K} \text { with } \operatorname{Tr}_{L}^{K}(a)=\sum_{g \in K / L} a^{g},
$$

for all $a \in A^{L}$, is called the relative trace map. In this sum $g$ runs through a set of representatives of $K / L$. For a $p$-subgroup $P$ in $G$ we denote by $A(P)$ the quotient

$$
A^{P} / \sum_{R<P} A_{R}^{P}
$$

and by $\operatorname{Br}_{P}$ the $N_{G}(P)$-algebra epimorphism

$$
A^{P} \rightarrow A(P) \text {. }
$$

Any $G$-interior algebra is naturally endowed with a $G \times G$-module structure given by

$$
\left(g_{1}, g_{2}\right) \cdot a=g_{1} \cdot a \cdot g_{2}^{-1}
$$

for any $g_{1}, g_{2} \in G$ and $a \in A$. If $A^{\prime}$ is another $G$-algebra a homomorphism of $G$ algebras is any homomorphism of $\mathcal{O}$-algebras $\psi: A \rightarrow A^{\prime}$ that verifies $\psi\left(a^{g}\right)=\psi(a)^{g}$, for any $a \in A^{\prime}$ and $g \in G$.

If $H$ is a normal subgroup of $G$, an $H$-interior $G$-algebra $A$ is an algebra simultaneously endowed with an $H$-interior and $G$-algebra structure with $G$ acting on the natural algebra homomorphism

$$
\mathcal{O} H \rightarrow A
$$

We refer the reader to [7] for the language of pointed groups and defect pointed groups on $G$-algebras. Throughout the proof of Theorem 4.2 we freely use the fact that, for any finite group $G$, the epimorphism of $G$-interior algebras

$$
\mathcal{O} G \rightarrow k G
$$

induces a defect group preserving bijection between the blocks of the two group algebras. Furthermore, if $G$ is normal in some finite group $\tilde{G}$ the epimorphism of $\tilde{G}$ algebras 2.1 also induces a defect group preserving bijection between the $\tilde{G}$-invariant blocks of $\mathcal{O} G$ and those of $k G$. We also frequently use the results of [1]. 


\section{The extended Brauer quotient}

Let $A$ be a $G$-interior algebra and consider $P$, a $p$ subgroup of $G$. For any $\varphi \in \operatorname{Aut}(P)$ we set $\Delta_{\varphi}(P): P \rightarrow P \times P$ the $\varphi$-twisted diagonal subgroup of $P \times P$ defined as

$$
\Delta_{\varphi}(P)=\{(u, \varphi(u)) \mid u \in P\} .
$$

Since $A$ is a $G \times G$-module we use the definition of $\Delta_{\varphi}(P)$ to introduce the $\mathcal{O}$-module

$$
A^{\Delta_{\varphi}(P)}=\{a \in A \mid(u, \varphi(u)) \cdot a=a \text { for all } u \in P\} .
$$

We briefly present here the construction of the extended Brauer quotient. Let $R$ be a subgroup of $P$, we also introduce the $\mathcal{O}$-module

$$
A_{\Delta_{\varphi}(R)}^{\Delta_{\varphi}(P)}=\left\{\operatorname{Tr}_{\Delta_{\varphi}(R)}^{\Delta_{\varphi}(P)}(a) \mid a \in A^{\Delta_{\varphi}(R)}\right\} .
$$

It is easily checked that $A^{\Delta_{\varphi}(P)} \cdot A^{\Delta_{\psi}(P)} \subseteq A^{\Delta_{\varphi \psi}(P)}$ for all $\varphi, \psi \in \operatorname{Aut}(P)$. Denote by $\varphi_{g} \in \operatorname{Aut}(P)$ the group homomorphism $\varphi_{g}(u)=g^{-1} u g$ for al $g \in N_{G}(P)$ and $u \in P$. Then the external direct sum

$$
N_{A}^{\operatorname{Aut}(P)}(P)=\bigoplus_{\varphi \in \operatorname{Aut}(P)} A^{\Delta_{\varphi}(P)}
$$

is an $N_{G}(P)$-interior algebra via $N_{G}(P) \ni g \mapsto g \cdot 1_{A} \in A^{\Delta_{\varphi_{g}}(P)}$, while the sum

$$
\mathrm{I}:=\bigoplus_{\varphi \in \operatorname{Aut}(P)}\left(\sum_{R<P} A_{\Delta_{\varphi}(R)}^{\Delta_{\varphi}(P)}\right)
$$

is a two-sided ideal of $N_{A}^{\operatorname{Aut}(P)}(P)$.

Definition 3.1. The extended Brauer quotient associated with the interior $G$-algebra $A$ and the $p$-group $P$ is the $N_{G}(P)$-interior algebra

$$
\begin{aligned}
\bar{N}_{A}^{\operatorname{Aut}(P)}(P): & =N_{A}^{\operatorname{Aut}(P)}(P) / \mathrm{I} \\
& =\bigoplus_{\varphi \in \operatorname{Aut}(P)} A^{\Delta_{\varphi}(P)} /\left(\sum_{R<P} A_{\Delta_{\varphi}(R)}^{\Delta_{\varphi}(P)}\right) \\
& =: \bigoplus_{\varphi \in \operatorname{Aut}(P)} A\left(\Delta_{\varphi}(P)\right)
\end{aligned}
$$

Remark 3.2. Definition 3.1 can be given for any subgroup of $\operatorname{Aut}(P)$. It was generalized to the case of $H$-interior $G$-algebras, where $H$ is normal in $G$ and $P \leq G$, in [4]. In this situation the authors restrict the constructions presented in 3.1 through 3.4 for all automorphisms $\varphi \in \operatorname{Aut}(P)$ that satisfy $\varphi(u) \in u H$ for all $u \in P$. Further generalizations of the extended Brauer quotient are given in [3]. We present them here. Assume $H$ is normal in $G$ and $B$ is an $H$-interior $G$-algebra. Then

$$
A:=B \otimes \mathcal{O H}_{H} \mathcal{O}
$$


is a $G$-interior algebra via $g \mapsto 1 \otimes g$ with the multiplication ginev by

$$
(a \otimes g)(b \otimes h)=a b^{g^{-1}} \otimes g h \text { for } a, b \in A \text { and } g h \in G .
$$

Obviously $A$ is a $\bar{G}:=G / H$-graded $G$-algebra with $A_{\bar{g}}:=B \otimes g$ for any $g \in \bar{g}$ and any $\bar{g} \in \bar{G}$. Furthermore if $x \in G$ and $\bar{g} \in \bar{G}$ then $\left(A_{\bar{g}}\right)^{x}=A_{\overline{g^{x}}}$. Set $\bar{P}=P H / H$. Any element $\varphi \in \operatorname{Aut}(P)$ determines an element $\bar{\varphi} \in \operatorname{Aut}(\bar{P})$ with $\bar{\varphi}(u)=\overline{\varphi(u)}$ for all $u \in P$. According to [3, Paragraph 4] quotient of the groups

$$
\begin{aligned}
\operatorname{Aut}_{\bar{G}}(P) & =\left\{\varphi \in \operatorname{Aut}(P) \mid \overline{\varphi(u)}=\overline{u^{g}} \text { for some } \bar{g} \in \bar{G} \text { and for all } u \in P\right\} \\
& \text { and } \\
\operatorname{Aut}_{\overline{1}}(P) & =\{\varphi \in \operatorname{Aut}(P) \mid \overline{\varphi(u)}=\bar{u} \text { for all } u \in P\}
\end{aligned}
$$

determine the graded structure of $\bar{N}_{A}^{\operatorname{Aut}(P)}(P)$. In the case $B=\mathcal{O} H$,

$$
\bar{N}_{A}^{\operatorname{Aut}(P)}(P) \simeq k N_{G}(P)
$$

as $N_{G}(P) / C_{H}(P)$-graded $N_{G}(P)$-interior algebras.

\section{A property of the generalized Brauer pairs}

We use the notations above, and chose $H$ a normal subgroup of $G$ and $b$ a block of $\mathcal{O} H$ that is $G$-invariant. Let $Q$ denote a $p$-subgroup of $G$ and consider the Brauer homomorphism with respect to $Q$

$$
\operatorname{Br}_{Q}: \mathcal{O} H^{Q} \rightarrow k C_{H}(Q)
$$

Let $\left(Q, e_{Q}\right)$ be a $(G, H, b)$-Brauer pair. That is $e_{Q}$ is a block of $k C_{H}(Q)$ that verifies $\operatorname{Br}_{Q}(b) e_{Q}=e_{Q}$. Since $C_{H}(Q)$ is a normal subgroup of $N_{G}(Q)$, the latter group acts on $Z\left(k C_{H}(Q)\right)$ hence it makes sense to consider the stabilizer of $e_{Q}$ in $N_{G}(Q)$, denoted $N_{G}\left(Q, e_{Q}\right)$. Consider the group homomorphism

$$
N_{G}(Q) \rightarrow \operatorname{Aut}(Q)
$$

Let $T$ denote a subgroup of the image of $N_{G}\left(Q, e_{Q}\right)$ in Aut $(Q)$. We denote by $N_{G}^{T}(Q)$ the inverse image of $T$ via 4.1 .

Finally we denote $N_{H}^{T}(Q)=N_{G}^{T}(Q) \cap H$ and $Q_{1}=Q \cap N_{H}(Q)$ and we assume that $Q_{1}$ is not trivial.

Remark 4.1. As $[6,4.1]$ states for the case $H=G$ any block of $k C_{H}(Q)$ remains a block of $k H$ where $C_{G}(Q) \leq H \leq N_{G}^{T}(Q)$. In our situation it might happen that a block of $k C_{H}(Q)$ is not primitive in $Z\left(k N_{H}^{T}(Q)\right)$. It could happen that is also not primitive in $k N_{H}^{T}(Q)^{N_{G}^{T}(Q)}$, unless $Q \leq N_{G}^{T}(Q)$. In any case the injective homomorphism 4.3 can be constructed and used for its purpose in the proof.

Theorem 4.2. There exists a point $\nu$ of $Q \cdot N_{G}^{T}(Q)$ on $\mathcal{O} H b$ with $\operatorname{Br}_{Q}(\nu)=e_{Q}$, and for any local pointed group $R_{\epsilon}$ on $\mathcal{O} H b$ satisfying $Q \leq R$ and $R_{\epsilon} \leq Q \cdot N_{G}^{T}(Q)_{\nu}$, there is a local pointed group $N_{R}^{T}(Q)_{\bar{\epsilon}}$ on $\mathcal{O} N_{H}^{T}(Q) e_{Q}$ such that there exists a $N_{R}^{T}(Q)$-interior algebra embedding

$$
\left(k N_{G}^{T}(Q)\right)_{\bar{\epsilon}} \rightarrow \bar{N}_{(\mathcal{O} G b)_{\epsilon}}^{T}(Q)
$$


determined by the structural homomorphism

$$
N_{G}^{T}(Q) \rightarrow \bar{N}_{\mathcal{O} G b}^{T}(Q) .
$$

Moreover, $R_{\epsilon}$ is a defect pointed group of $Q \cdot N_{G}^{T}(Q)_{\nu}$ on $\mathcal{O} H b$ if and only if $N_{R}^{T}(Q)_{\mathrm{Br}_{Q}(\epsilon)} \leq N_{G}^{T}(Q)_{\left\{e_{Q}\right\}}$ is a maximal local pointed group with this property over $k C_{H}(Q) e_{Q}$. In particular, $N_{R}^{T}(Q)$ is a defect group of $e_{Q}$ as a primitive idempotent of $\left(k C_{H}(Q)\right)^{N_{G}^{T}(Q)}$ if and only if $R$ is a defect group of $e_{Q}$ as a primitive idempotent of $\left(k\left(Q_{1} \cdot N_{H}^{T}(Q)\right)^{Q \cdot N_{G}^{T}(Q)}\right.$.

Proof. The block $e_{Q}$ lies in $k C_{H}(Q)^{Q \cdot N_{G}^{T}(Q)}$, where it remains primitive. It is clear that it lifts to a point $\nu \subseteq(\mathcal{O} H)^{Q \cdot N_{G}^{T}(Q)}$ via $\operatorname{Br}_{Q}$. Since $\operatorname{Br}_{Q}(b) e_{Q}=e_{Q}$ we obtain $b \nu=\nu$ giving $\nu \subseteq(\mathcal{O} H b)^{Q \cdot N_{G}^{T}(Q)}$. For any $T$ chosen as above $e_{Q}$ is a primitive idempotent in $\mathcal{O} N_{H}^{T}(Q)^{Q \cdot N_{G}^{T}(Q)}$ and in $\mathcal{O}\left(Q_{1} \cdot N_{H}^{T}(Q)\right)^{Q \cdot N_{G}^{T}(Q)}$ according to [8, Lemma 2.1].

Let $R_{\epsilon}$ be as mentioned. Since $Q$ is normal in $R$ and since we deal with permutation algebras we get that $\operatorname{Br}_{R}^{\mathcal{O} H}(\epsilon) \neq 0$ implies $\operatorname{Br}_{Q}^{\mathcal{O} H}(\epsilon) \neq 0$. Furthermore $\operatorname{Br}_{Q}(\epsilon) e_{Q}=\operatorname{Br}_{Q}(\epsilon)$, we set $\tilde{\epsilon}=\operatorname{Br}_{Q}(\epsilon)$ and since $\operatorname{Br}_{R}^{k C_{H}(Q)}(\tilde{\epsilon}) \neq 0$ it is clear that $N_{R}^{T}(Q)_{\tilde{\epsilon}}$ is a local pointed group on $k C_{H}(Q) e_{Q}$. The injective algebra homomorphism

$$
\left(k C_{H}(Q) e_{Q}\right)\left(N_{R}^{T}(Q)\right) \rightarrow\left(k N_{H}^{T}(Q) e_{Q}\right)\left(N_{R}^{T}(Q)\right)
$$

determines a point $\bar{\epsilon} \subseteq\left(k N_{H}^{T}(Q) e_{Q}\right)^{N_{R}^{T}(Q)}$ satisfying

$$
\tilde{\epsilon} \cdot \bar{\epsilon}=\bar{\epsilon} \text { and } \operatorname{Br}_{N_{R}^{T}(Q)}^{k N_{H}^{T}(Q)}(\bar{\epsilon}) \neq 0 .
$$

The construction of the extended Brauer quotient and [4, Theorem 3.1] give the $N_{R}^{T}(Q)$-interior algebra isomorphism

$$
\bar{N}_{(\mathcal{O} G b)_{\epsilon}}^{T}(Q) \simeq\left(\bar{N}_{\mathcal{O} G b}^{T}(Q)\right)_{\tilde{\epsilon}} .
$$

We apply [3, Remark 2.4] and 4.4 to obtain that the group homomorphism

$$
N_{G}^{T}(Q) \rightarrow\left(\bar{N}_{\mathcal{O} G b}^{T}(Q)\right)^{*},
$$

given by

$$
g \mapsto \overline{g \cdot b} \in\left(\overline{(\mathcal{O} G b)^{\Delta_{\varphi_{g}}(Q)}}\right)^{*}
$$

induces the mentioned $N_{R}^{T}(Q)$-interior algebra embedding.

First assume that $R_{\epsilon}$ is a defect pointed group of $Q \cdot N_{G}^{T}(Q)_{\nu}$ on $\mathcal{O} H b$.

Then $\nu \subseteq(\mathcal{O H b})_{R}^{Q \cdot N_{G}^{T}(Q)}$ and $\nu \cdot \epsilon=\epsilon$. Applying $\operatorname{Br}_{Q}$ on the last equality we get $e_{Q} \cdot \operatorname{Br}_{Q}(\epsilon)=\operatorname{Br}_{Q}(\epsilon)$ and $e_{Q} \in k C_{H}(Q)_{N_{R}^{T}(Q)}^{N_{T}^{T}(Q)}$. According to 4.4 we obtain $\operatorname{Br}_{N_{R}^{T}(Q)}^{k C_{H}(Q)}\left(e_{Q}\right) \neq 0$.

Conversely, by our assumptions we have $e_{Q} \cdot \operatorname{Br}_{Q}(\epsilon)=\operatorname{Br}_{Q}(\epsilon), e_{Q} \in k C_{H}(Q)_{N_{R}^{T}(Q)}^{N_{T}^{T}(Q)}$, $\operatorname{Br}_{N_{R}^{T}(Q)}^{k C_{H}(Q)}\left(e_{Q}\right) \neq 0$, for some local point $\epsilon \subseteq(\mathcal{O} H b)^{Q \cdot N_{R}^{T}(Q)}$. Using the $\mathcal{O}$-algebra epimorphism

$$
(\mathcal{O} H b)_{Q \cdot N_{R}^{T}(Q)}^{Q \cdot N_{T}^{T}(Q)} \rightarrow\left(k C_{H}(Q) e_{Q}\right)_{Q \cdot N_{R}^{T}(Q)}^{Q \cdot N_{T}^{T}(Q)}
$$


we see that $\nu$ in relatively $R=Q \cdot N_{R}^{T}(Q)$-projective and $\nu \cdot \epsilon=\epsilon$. This concludes the proof of the first equivalence.

We use [8, Lemma 2.1] again to obtain that $e_{Q} \in k\left(Q_{1} \cdot N_{H}^{T}(Q)\right)^{Q \cdot N_{G}^{T}(Q)}$ is still a primitive idempotent. The last assertion follows immediately from the above statements by using $Q_{1} \cdot N_{H}^{T}(Q)$ in place of $H$ and $e_{Q}$ in place of $\nu$.

Remark 4.3. Although the block $e_{Q}$ is not necessarily primitive in $Z\left(k N_{H}^{T}(Q)\right)$ or in $k N_{H}^{T}(Q)^{N_{G}^{T}(Q)}$, the theorem above covers [6, Lemma 4.18] when applied in the case $H=G$, which gives $Q_{1}=Q$, and by substituting $C_{G}(Q)$ for $N_{G}^{T}(Q)$ in the second statemen of the theorem. We can obtain another generalization as it follows from the next corollary.

Corollary 4.4. There exists a point $\nu$ of $Q \cdot N_{G}^{T}(Q)$ on $\mathcal{O} H b$ with $\operatorname{Br}_{Q}(\nu)=e_{Q}$ and for any local pointed group $R_{\epsilon}$ on $\mathcal{O} H b$ satisfying $Q \leq R$ and $R_{\epsilon} \leq Q \cdot N_{G}^{T}(Q)_{\nu}$, there is a local pointed group $N_{R}^{T}(Q)_{\bar{\epsilon}}$ on $k C_{H}(Q) e_{Q}$ such that there exists a $N_{R}^{T}(Q)$-interior $N_{G}^{T}(Q) / C_{H}(Q)$-graded algebra isomorphism

$$
\left(k N_{G}^{T}(Q)\right)_{\bar{\epsilon}} \rightarrow \bar{N}_{(\mathcal{O} G b)_{\epsilon}}^{T}(Q)
$$

determined by the structural homomorphism

$$
N_{G}^{T}(Q) \rightarrow \bar{N}_{\mathcal{O} G b}^{T}(Q) .
$$

Moreover, $R_{\epsilon}$ is a defect pointed group of $Q \cdot N_{G}^{T}(Q)_{\nu}$ on $\mathcal{O} H b$ if and only if $N_{R}^{T}(Q)_{\operatorname{Br}_{Q}(\epsilon)} \leq N_{G}^{T}(Q)_{\left\{e_{Q}\right\}}$ is a maximal local pointed group with this property over $k C_{H}(Q) e_{Q}$. In particular, $N_{R}^{T}(Q)$ is a defect group of $e_{Q}$ as a primitive idempotent of $\left(k C_{H}(Q)\right)^{N_{G}^{T}(Q)}$ if and only if $R$ is a defect group of $e_{Q}$ as a primitive idempotent of $\left(k Q_{1} \cdot C_{H}(Q)\right)^{Q \cdot N_{G}^{T}(Q)}$.

Remark 4.5. 1. Notice that we can also consider $T$ such that $N_{G}^{T}(Q)=$ $N_{G}\left(Q, e_{Q}\right)$, but in this situation the statement of Corollary 4.4 only points out the $N_{G}\left(Q, e_{Q}\right) / C_{H}(Q)$-graded isomorphism 4.5 and the fact that the primitive idempontents of $k C_{H}(Q)^{N_{G}\left(Q, e_{Q}\right)}$ are the primitive idempotents of $\left(k Q_{1} C_{H}(Q)\right)^{N_{G}\left(Q, e_{Q}\right)}$ admitting the same defect group $R$ that includes $Q$.

2. [2, Lemma 6.6] is an important ingredient of the proof of the main result $[2$, Theorem 6.9], and it can be deduced from the above corollary when applied in the general situation. Indeed, if $T$ is a defect group of $e_{Q}$ as a primitive idempotent of $k C_{H}(Q)^{N_{G}^{K}(Q)}$ then $Q \cdot T$ is a defect group of $\nu$ as a primitive idempotent of $\mathcal{O} H^{Q \cdot N_{G}^{K}(Q)}$. At this point we denote by $\tilde{e}_{Q}$ the primitive idempotent of $\mathcal{O} C_{H}(Q)^{N_{G}^{K}(Q)}$ corresponding to $e_{Q}$ via 2.1. Note that $\tilde{e}_{Q}$ is primitive in $\mathcal{O} C_{H}(Q)^{Q \cdot N_{G}^{K}(Q)}$ and $\operatorname{Br}_{Q}\left(\tilde{e}_{Q}\right)=e_{Q}$. Consider the decomposition

$$
\mathcal{O} H^{Q \cdot N_{G}^{K}(Q)}=\mathcal{O} C_{H}(Q)^{Q \cdot N_{G}^{K}(Q)} \bigoplus\left(\sum_{Q^{\prime}<Q}(\mathcal{O} H)_{Q^{\prime}}^{Q}\right)^{Q \cdot N_{G}^{K}(Q)}
$$

Since the sum on the right lies in the radical of $\mathcal{O} H$ it follows that $\nu \subseteq$ $\mathcal{O} C_{H}(Q)^{Q \cdot N_{G}^{K}(Q)}$ and then $\nu=\tilde{e}_{Q}$. This means that $e_{Q}$ has defect group $Q \cdot \bar{T}$ 
as a primitive idempotent of $k C_{H}(Q)^{Q \cdot N_{G}^{K}(Q)}$. At last, if $Q \subseteq N_{G}^{K}(Q)$ then up to a $N_{G}(Q)$-conjugate we have $Q \cdot T=T$ forcing $Q \subseteq T$.

\section{References}

[1] Broué, M., Puig, L., Characters and local structure in G-algebras, J. Algebra, 63 (1980), 306-317.

[2] Coconeţ, T., Marcus, A., Group graded basic Morita equivalences, J. Algebra, 489(2017), 1-24.

[3] Coconet,, T., Marcus, A., Remarks on the extended Brauer quotient, J. Algebra, 491(2017), 78-89.

[4] Coconet, T., Todea, C.-C., The extended Brauer quotient of $N$-interior $G$-algebras, J. Algebra, 396(2013), 10-17.

[5] Puig, L., Zhou, Y., A local property of basic Morita equivalences, Math. Z., 256(2007), 551-562.

[6] Puig, L., Zhou, Y., A local property of basic Rickard equivalences, J. Algebra, 322(2009), 1946-1973.

[7] Thévenaz, J., G-Algebras and Modular Representation Theory, Clarendon Press, Oxford, 1995.

[8] Todea, C.-C., On some saturated triples and hyperfocal subgroups, J. Algebra, 499 (2018), 358-374.

Tiberiu Coconet

Babeş-Bolyai University,

Faculty of Economics and Business Administration,

58-60, Teodor Mihali Street,

400591 Cluj-Napoca, Romania

Department of Mathematics,

Technical University of Cluj-Napoca,

Str. G. Bariţiu 25,

Cluj-Napoca 400027, Romania

e-mail: tiberiu. coconet@math . ubbcluj.ro 